\title{
OSCILLATION PROPERTIES OF TWO TERM LINEAR DIFFERENTIAL EQUATIONS
}

\author{
BY \\ G. A. BOGAR( $\left.{ }^{1}\right)$
}

\begin{abstract}
The two term differential equations $L_{n}[y]+p y=0$, where $L_{0}[y]=y$, $L_{i}[y]=\left(\rho_{i}(t) L_{i}[y(t)]\right)^{\prime}$, were recently studied by Z. Nehari. In this paper we give integral conditions which assure the integrability of $\rho_{1}^{-1}(t) p(t)$ on $[a, \infty)$ when $L_{n}[y]$ is disconjugate. By changing the integral conditions slightly we then prove that the equation has $n$ linearly independent oscillatory solutions.
\end{abstract}

1. Introduction. The differential equations, which will be considered here, are of the form

$$
L_{n}[y]+p y=0,
$$

where $L_{0}[y]=y, L_{i}[y]=\left(\rho_{i} L_{i-1}[y]\right)^{\prime}, \quad i=1, \ldots, n$, and $\rho_{i}(x)$ are positive and continuous in $(0, \infty)$. Also $p(x)$ is continuous in $(0, \infty)$.

A function $y$ will be considered as a solution of (1.1) if $L_{j}[y], j=0, \ldots, n$, exists and is continuous and $y$ satisfies (1.1).

Definition 1.1. (a) Equation (1.1) is said to be disconjugate on $[a, \infty)$ if no nontrivial solution of (1.1) has more than $n-1$ zeros, counting multiplicities, on $[a, \infty)$.

(b) If $y$ is a solution of $(1.1), y$ is said to be oscillatory on $[a, \infty)$ provided it has infinitely many zeros on $[a, \infty)$.

Definition 1.2. For $t \in[a, \infty)$ define $\eta_{1}(t)$ to be the greatest lower bound of the set of all $b>t$ such that (1.1) has a nontrivial solution with at least $n$ zeros on $[t, b] ; \eta_{1}(t)$ is called the first conjugate point of $t$.

Definition 1.3. A fundamental set of solutions $\left\{u_{\beta}(x, t)\right\}$ of $(1.1)$ is defined by

$$
L_{\alpha}\left[u_{\beta}(t, t)\right]=\delta_{\alpha, \beta}, \quad(\alpha, \beta=0, \ldots, n-1) .
$$

In $\$ 2$ we prove a theorem which gives Theorem I of Z. Nehari [5] with less conditions and Theorem 11.2 of W. Leighton and Z. Nehari [2] for equation (1.1). $\S 3$ is devoted to conditions under which there exist $n$ oscillatory solutions to (1.1).

Received by the editors July 16, 1970 and, in revised form, January 5, 1971.

AMS 1970 subject classifications. Primary 34C10; Secondary 34B10.

Key words and phrases. Nonoscillatory equations, disconjugate, oscillatory solution, fundamental set of solutions, iterated integrals, Green's function.

(1) Part of the results of this paper were part of the author's dissertation. The author wishes to thank Professor J. S. Bradley for his advice and assistance. The work was supported, in part, by NASA Grant Number NGR 43-001-029.

Copyright (C) 1971, American Mathematical Society 
2. First let us develop some notation which will be used in the remainder of the paper.

$$
\begin{gathered}
\varphi_{k}(x, a)=\int_{a}^{x} \frac{d t_{1}}{\rho_{2}\left(t_{1}\right)} \int_{a}^{t_{1}} \frac{d t_{2}}{\rho_{3}\left(t_{2}\right)} \cdots \int_{a}^{t_{k-2}} \frac{d t_{k-1}}{\rho_{k}\left(t_{k-1}\right)} \\
\psi_{k}(x, a)=\int_{a}^{x} \frac{d t_{1}}{\rho_{k}\left(t_{1}\right)} \int_{a}^{t_{1}} \frac{d t_{2}}{\rho_{k+1}\left(t_{2}\right)} \cdots \int_{a}^{t_{n-k}} \frac{d t_{n-k+1}}{\rho_{n}\left(t_{n-k+1}\right)}, \quad k=\{2, \ldots, n\},
\end{gathered}
$$

and

$$
S(x, a)=\int_{a}^{x} \frac{d t_{1}}{\rho_{n}\left(t_{1}\right)} \int_{t_{1}}^{x} \frac{d t_{2}}{\rho_{n-1}\left(t_{2}\right)} \cdots \int_{t_{n-1}}^{x} \frac{d t_{n-1}}{\rho_{2}\left(t_{n-1}\right)}
$$

THEOREM 2.1. Let $p(x)$ and $\rho_{i}(x)(i=1, \ldots, n)$ be continuous and positive on $[a, \infty)$. Suppose that $\psi_{k}(x, a) \rightarrow \infty$ as $x \rightarrow \infty(k=2, \ldots, n-1)$. If the differential equation (1.1) is disconjugate on $[a, \infty)$, then $L_{i}\left[u_{n-1}(x, a)\right] \geqq 0$ on $[a, \infty)$ where $i=\{0, \ldots, n-1\}$.

Proof. Let $y=u_{n-1}(x, a)$. If, for some $i \in\{1, \ldots, n-1\}, L_{i}[y]$ has more than one simple zero on $(a, \infty)$, then by repeated application of Rolle's Theorem, we see that $L_{n}[y]$ must have a zero on $(a, \infty)$, but this is a contradiction.

Assume $L_{1}[y]$ has a zero on $(a, \infty)$, say at $x=c_{1}$; then by Rolle's Theorem $L_{i}[y]$, $i \in(1,2, \ldots, n-1)$, has a zero on $\left(a, c_{1}\right)$. Since $u_{n-1}(x, a) \equiv y(x)$, we know that all the functions $L_{i}[y]$, for $1 \leqq i \leqq n-1$, are negative on $\left[c_{1}, \infty\right)$. Also $L_{n}[y]=-p y$ so that $L_{n}[y]<0$ on $\left[c_{1}, \infty\right)$ and

$$
\int_{c_{1}}^{x} L_{n}[y]=-\int_{c_{1}}^{x} p y<0
$$

therefore

$$
-\rho_{n} L_{n-1}[y](x)>-\left.\rho_{n} L_{n-1}[y(x)]\right|_{x=c_{1}}=\tilde{c}>0
$$

or

$$
-L_{n-1}[y](x)=\tilde{c} / \rho_{n}(x)>0 .
$$

Thus after integrating $n$ times, we have

$$
-\rho_{1} y(x)+\rho_{1} y\left(c_{1}\right) \geqq \tilde{c} \psi_{2}\left(x, c_{1}\right)>0 .
$$

Now

$$
\lim _{x \rightarrow \infty}\left(\left(-\rho_{1} y\right)(x)+\rho_{1} y\left(c_{1}\right)\right) \geqq \infty,
$$

but this is a contradiction.

By using a similar argument and the hypothesis that $\psi_{i}(x, a) \rightarrow \infty$, we can show that $L_{i}[y] \geqq 0$ on $[a, \infty)$ and $L_{i}[y]>0$ on $(a, \infty), i=1, \ldots, n-1$.

Using Theorem 2.1, we can now prove Theorem I of Z. Nehari [5] with the hypothesis that $\varphi_{k}(x, a) \rightarrow \infty$ as $x \rightarrow \infty, k \in\{2, \ldots, n-1\}$, deleted. 
THEOREM 2.2. Let $p(x)$ and $\rho_{i}(x)(i=1, \ldots, n)$ be continuous and positive on $[a, \infty)$. Suppose $\psi_{k} \rightarrow \infty$ as $x \rightarrow \infty$ for $k=2, \ldots, n-1$. If the differential equation (1.1) is disconjugate in $[a, \infty)$, then $\left[\rho_{1}(x)\right]^{-1} p(x)$ is integrable over this interval and

$$
\rho_{1}(x) S(x, a) \int_{x}^{\infty} \rho_{1}^{-1} p d x \leqq 1 .
$$

The hypothesis that $\psi_{k} \rightarrow \infty$ as $x \rightarrow \infty, k=2, \ldots, n-1$, leads also to a generalization of a theorem of Leighton and Nehari [2] for $y^{(\mathrm{IV})}+p y=0$ and Leslie [3] for $y^{(2 n)}+p y=0$.

LEMMA 2.1. If $y$ satisfies equation (1.1) and $y(x)>0$ for $x \in(b, \infty), b \geqq a$, and $\psi_{k}(x, a) \rightarrow \infty$ as $x \rightarrow \infty, k=\{2, \ldots, n\}$, then $L_{n-1} y(x)>0$ for $x \in(b, \infty)$.

Proof. Assume $L_{n-1} y$ has a zero on $(b, \infty)$, say at $x=c$, then $L_{n-1} y(x)<0$ on $\left(c_{1}, \infty\right), c_{1}>c$, since $L_{n-1} y(x)$ can have at most one zero on $(b, \infty)$.

$$
\int_{c_{1}}^{x} L_{n}[y]=\rho_{n} L_{n-1} y(x)=\rho_{n} L_{n-1} y\left(c_{1}\right)<0
$$

or

$$
-L_{n-1}[y](x)=-\rho_{n} L_{n-1} y\left(c_{1}\right) / \rho_{n}(x)>0
$$

Now

$$
-\rho_{n-1} L_{n-2}[y(x)]+\rho_{n-1} L_{n-2} y\left(c_{2}\right)=\int_{c_{1}}^{x} \frac{\tilde{c}}{\rho_{n}(t)} d t
$$

and

$$
\lim _{x \rightarrow \infty}\left[-\rho_{n-1} L_{n-2} y[x]+\rho_{n} L_{n-2} y\left(c_{2}\right)\right]=\infty
$$

which implies $L_{n-2}[y(x)] \rightarrow-\infty$ as $x \rightarrow \infty$. Thus, there exists a $c_{2}$ such that

$$
L_{n-2}[y(x)]<0 \text { on }\left[c_{2}, \infty\right) \text {. }
$$

Now

$$
-\rho_{n-1} L_{n-2}[y(x)]+\rho_{n-1} L_{n-2} y\left(c_{2}\right)=\int_{c_{2}}^{x} \frac{\tilde{c}}{\rho_{n}(t)} d t
$$

and

$$
-\rho_{n-1} L_{n-2} y(x)>\int_{c_{2}}^{x} \frac{\tilde{c}}{\rho_{n}(t)} d t .
$$

Using this process and $\psi_{k}(x, a) \rightarrow \infty$ for $x \rightarrow \infty, k=(2, \ldots, n-1)$, we arrive finally at

$$
-\rho_{n} y(x)+\rho_{1} y\left(c_{n-1}\right)>\tilde{c} \psi_{2}\left(x, c_{n-1}\right)>0
$$

Hence

$$
\lim _{x \rightarrow \infty}\left(\left(-\rho_{1} y\right)(x)+\rho_{1} y\left(c_{n-1}\right)\right) \geqq \infty,
$$

but this is a contradiction. 
LEMMA 2.2. If (1.1) is nonoscillatory and $y$ is a solution of (1.1), $y$ is a solution of $(1.1)$ which is positive on $(a, \infty)$. Also suppose $\psi_{k}(x, a) \rightarrow \infty$ as $x \rightarrow \infty$, then

$$
-\infty<\lim _{x \rightarrow \infty} \frac{y(x)}{T_{n}(x, a)}=\lim _{x \rightarrow \infty} \rho_{n} L_{n-1} y(x)<\infty
$$

when $T_{k}(x, a)=\left(1 / \rho_{1}\right) \varphi_{k}(x, a), k=2, \ldots, n$, and $T_{1}(x, a)=1 / \rho_{1}$.

Proof. After $n-1$ integrations by parts of (1.1), we can write (1.1) as

$$
R(x, a)=y(x)+\int_{a}^{x} T_{n}(x, t) p(t) y(t) d t
$$

where $R(x, a)=\sum_{k=0}^{n-1} \rho_{k+1}\left[L_{k} y\right]_{x=a} T_{k+1}(x, a)$. Since $y(x)>0$, then

$$
R(x, a) \leqq y(x)+T_{n}(x, a) \int_{a}^{x} p y d t .
$$

Thus,

$$
\frac{R(x, a)}{T_{n}(x, a)} \leqq \frac{y(x)}{T_{n}(x, a)}+\rho_{n} L_{n-1} y(a)-\rho_{n} L_{n-1}(x)
$$

Now

$$
\frac{T_{k}(x, a)}{T_{n}(x, a)}<\frac{T_{k}(x, a)}{T_{k}(x, \alpha)} \frac{1}{\psi_{k+1}(\alpha, a)}=\frac{\varphi_{k}(x, a)}{\varphi_{k}(x, \alpha)} \frac{1}{\psi_{k+1}(\alpha, a)} .
$$

If $\varphi_{k}(x, a) \rightarrow \infty$ as $x \rightarrow \infty$, then $\lim _{x \rightarrow \infty} \varphi_{k}(x, a) / \varphi_{k}(x, \alpha)=1$; hence $T_{k}(x, a) / T_{n}(x, a)$ $\rightarrow 0, k=2, \ldots, n-1$. On the other hand, if $\varphi_{k}(x, a) \rightarrow k$ as $x \rightarrow \infty$, then $T_{k}(x, a) / T_{n}(x, a) \rightarrow 0, k=2, \ldots, n-1$. Hence

$$
\lim _{x \rightarrow \infty} \frac{R(x, a)}{T_{n}(x, a)}=\left.\rho_{n} L_{n-1}[y]\right|_{x=a} .
$$

Because of Lemma 2.1 and $L_{n}[y]<0$, the $\lim _{x \rightarrow \infty} L_{n-1} y(x)$ exists. Combining (2.5) and (2.6)

$$
\lim _{x \rightarrow \infty} p_{n}(x) L_{n-1} y(x) \leqq \liminf _{x \rightarrow \infty} \frac{y(x)}{T_{n}(x, a)}
$$

From (2.4) if $a<\varepsilon<x$, we can obtain

$$
\frac{R(x, a)}{T_{n}(x, a)} \geqq \frac{y(x)}{T_{n}(x, a)}+\frac{\varphi_{n}(x, \varepsilon)}{\varphi_{n}(x, a)}\left[\rho_{n} L_{n-1} y(a)-\rho_{n} L_{n-1} y(\varepsilon)\right] .
$$

Since $0<\lim _{x \rightarrow \infty} \varphi_{n}(x, \varepsilon) / \varphi_{n}(x, a) \leqq 1$, we have by (2.6) for a fixed $\varepsilon$

$$
\rho_{n} L_{n-1} y(\varepsilon) \geqq \limsup _{x \rightarrow \infty} \frac{y(x)}{T_{n}(x, a)} .
$$

Since $L_{n-1} y$ is decreasing and the $\varepsilon$ above was arbitrary, we have

$$
\lim _{x \rightarrow \infty} \rho_{n} L_{n-1} y(x) \geqq \limsup _{x \rightarrow \infty} \frac{y(x)}{T_{n}(x, a)} .
$$


THEOREM 2.3. Suppose $\psi_{k}(x, a) \rightarrow \infty$ as $x \rightarrow \infty$, then (1.1) has a principal solution $y(x, a)$ such that

$$
\lim _{x \rightarrow \infty} T_{n}^{-1}(x, a)|y(x)|=c \quad(0<c<\infty)
$$

if and only if $\int_{a}^{\infty} T_{n}(x, a) p(x) d x<\infty(0<a<\infty)$.

Proof. In order to prove the sufficiency, we first choose $a$ large enough so that

$$
\int_{a}^{\infty} T_{n}(x, a) p(x) d x<\frac{1}{2}
$$

Now let $y(x, a)=u_{n-1}(x, a)$ and assume that $\eta_{1}(a)<\infty$. Using equation (2.4) the assumption that $\eta_{1}(a)<\infty$ leads to the contradiction

$$
1 \leqq \int_{a}^{\eta_{1}(a)} T_{n}(t, a) p(t) d t<\frac{1}{2}
$$

Thus $\eta_{1}(a)=\infty$ and therefore, by Lemma $2.2, \lim _{x \rightarrow \infty} y(x) / T_{n}(x, a)$ exists.

Using equation (2.4), we see that $T_{n}(x, a) \geqq y(x)$ and that

$$
T_{n}(x, a) \leqq y(x)+T_{n}(x, a) \int_{a}^{x} T_{n}(t, a) p(t) d t .
$$

Hence $T_{n}(x, a)<y(x)+T_{n}(x, a) / 2$ or $\frac{1}{2}<y(x) / T_{n}(x, a) \leqq 1$.

Since the $\lim _{x \rightarrow \infty} y(x) / T_{n}(x, a)$ exists and $\frac{1}{2}<y(x) / T_{n}(x, a) \leqq 1$, the sufficiency is proven.

The necessary part is a direct generalization of the proof given by Leighton and Nehari [2] for $y^{(\mathrm{IV})}+p y=0$.

For the equation

$$
L[y]=y^{(n)}+p y=0 \quad \text { with } p \geqq 0 \text { and } n \geqq 2,
$$

we obtain the following corollary:

COROLlaRY 2.1. Equation (2.7) has a principal solution $y(x)=y(x, a)$ such that

$$
\lim _{x \rightarrow \infty} x^{-n+1}|y(x)|=c \quad(0<c<\infty)
$$

if and only if $\int_{a}^{\infty} x^{n-1} p(x) d x<\infty(0<a<\infty)$.

3. Conditions for oscillation of equation (1.1). Before proving the main results of this section we prove a lemma and develop some notation.

LEMMA 3.1. If the differential equation (1.1) is nonoscillatory on $[a, \infty)$, then the zeros in $(a, \infty)$ of $L_{i}\left[u_{n-1}(x, a)\right]$ are at most simple for $0 \leqq i \leqq n-1$. Moreover, between the zeros of $u_{n-1}(x, a)$ in $(a, \infty)$, there exists one and only one zero of $L_{i}\left[u_{n-1}\right], 0 \leqq i \leqq n-1$. 
Proof. Since $L[y]=0$ is nonoscillatory on $[a, \infty)$, then there exists a $c \geqq a$ such that $u_{n-1}(x, a) \neq 0$ on $(c, \infty)$. From Theorem 2 of Mikusinki [4] we know that $u_{n-1}(x, a)$ has at most only simple zeros on $(a, \infty)$. If $u_{n-1}(x, a)>0$ for $x \in(a, \infty)$, then using Rolle's Theorem we see that the theorem follows. When $c>a$, let $m$ be the number of zeros on $(a, c]$. Now by Rolle's Theorem we can show that the zeros of $L_{i}\left[u_{n-1}\right]$ are simple. Let $c_{1}$ be the first zero of $u_{n-1}$ to the right of $a$. Again by Rolle's Theorem we can show that $L_{i}\left[u_{n-1}(x, a)\right]$ has one and only one zero in $\left(a, c_{1}\right]$ and the zero does not occur at $c_{1}$. After $m$ such applications the theorem follows.

Now let us develop some notation in order to simplify the statement of the next theorem.

$$
\chi_{i}(x, c)=\int_{c}^{x} \frac{d t}{\rho_{1+i}} \int_{c}^{t_{1}} \cdots \int_{c}^{t_{n_{1}-2}} \frac{d t_{n_{1}-1}}{\rho_{n_{1}}\left(t_{n_{1}-1}\right)} \text { for } 1 \leqq i \leqq n_{1}-1 .
$$

(b) For $n$ an integer, let $S$ be the set of all $j$ between 1 and $n_{1}-1$ when $n=2 n_{1}$ and the set of all $j$ between 1 and $n_{1}$ when $n=2 n_{1}+1$.

$$
\sigma_{j}(x, c)=\int_{c}^{x} \frac{d t}{\rho_{n_{1+1}+j}} \cdots \int_{c}^{t_{n_{1}-2}} \frac{d t_{n_{1}-1}}{\rho_{n}\left(t_{n_{1}-1}\right)} \text { for } j \in S .
$$

THEOREM 3.1. For $n>3$, where $n=2 n_{1}+1$ or $n=2 n_{1}$, suppose the following hold:

$$
\begin{gathered}
\chi_{i}(x, a) \rightarrow \infty \text { as } x \rightarrow \infty \text { for } 1 \leqq i \leqq n_{1}-1 ; \\
\sigma_{j}(x, a) \rightarrow \infty \text { as } x \rightarrow \infty \text { for } j \in S ; \\
\int_{a}^{\infty} \frac{d t}{\rho_{n_{1}+1}} \int^{t_{1}} \cdots \int^{t_{n_{1}-1}} \frac{d t_{n_{1}}}{\rho_{n}} \int^{t_{n_{1}}} \frac{p\left(t_{n_{1}+1}\right)}{\rho_{1}\left(t_{n_{1}+1}\right)} d t_{n_{1}+1}=\infty ; \\
\int_{a}^{\infty} T_{n-1}(x, a) p d x=\infty
\end{gathered}
$$

Then there exists a set of $n$ linearly independent oscillatory solutions of (1.1) and the zeros of one oscillatory solution separate the zeros of the other oscillatory solutions.

Proof. Assume that the theorem is false and consider the solution $y(x)$ $=c u_{n-1}(x, a)$, which has a zero of order $n-1$ at $a$. Let $b$ be the last zero of $y$ on $[a, \infty)$ and let $m$ be the number of zeros of $y$ on $(a, \infty)$. Since the zeros of $y$ are simple, let us assume without loss of generality that $y(x)>0$ on $(b, \infty)$. If $m$ is even then $y(x)>0$ between $a$ and the first zero of $y(x)$ on $(a, b]$; if $m$ is odd then $y(x)<0$ between $a$ and the first zero. Furthermore $L_{1}[y], \ldots, L_{n-1}[y]$ are all positive at $x=b$ (unless $b=a$ in which case the functions are all positive in a right-hand neighborhood of $x=a$ ), since the zeros of $L_{i}[y]$ are simple and occur between the zeros of $y$.

We now want to show that the functions $L_{1}[y], \ldots, L_{n_{1}-2}[y]$ do not have a zero on $(b, \infty)$ and hence are positive there. Assume that $L_{1}[y]$ has a zero at $x=c$ 
on $(b, \infty)$. By repeated use of Rolle's Theorem, each $L_{2}[y], \ldots, L_{n-1}[y]$ has one and only one zero on $(b, c)$ and no zeros on $[c, \infty)$. Thus, on $(c, \infty)$ they are all negative.

Since $L_{n_{1}}[y]=\left(\rho_{n_{1}} L_{n_{1}-1}(y)\right)^{\prime}<0$ or $-L_{n_{1}}[y]>0$, integrating from $x_{1} \in(c, x)$ to $x$ yields

$$
-\rho_{n_{1}} L_{n_{1}-1}[y(x)]+\rho_{n_{1}} L_{n_{1}-1}\left[y\left(x_{1}\right)\right]>0
$$

or

$$
-L_{n_{1}-1}[y(x)]>\frac{-\rho_{n_{1}} L_{n_{1}-1}\left[y\left(x_{1}\right)\right]}{\rho_{n_{1}}(x)}=\frac{c_{1}}{\rho_{n}(x)}>0 .
$$

By successive integration we arrive at

$$
-\rho_{1} y(x)+\rho_{1} y\left(x_{1}\right)>c_{1} \int_{x_{1}}^{x} \frac{d t}{\rho_{2}} \cdots \int_{x_{1}}^{t_{n_{1}-2}} \frac{d t_{n_{1}-1}}{\rho_{n_{1}}\left(t_{n_{1}-1}\right)}
$$

Hence $\lim _{x \rightarrow \infty}\left(-\rho_{1} y(x)+\rho_{1} y\left(x_{1}\right)\right)=\infty$, which implies that $\rho_{1} y(x) \rightarrow-\infty$, but this is a contradiction. Continuing the above process and using the hypothesis that $\chi_{i} \rightarrow \infty$ as $x \rightarrow \infty$, we see that $L_{i}[y(x)]>0\left(i=1, \ldots, n_{1}-1\right)$. Since $\rho_{1} y>0$ and $\left(\rho_{1} y\right)^{\prime}>0$ on $[b, \infty), \rho_{1} y$ is increasing.

Now assume there is a point $c$ such that $L_{n_{1}}[y]$ is negative on $[c, \infty)$. From the discussion above we know that $\rho_{1} y(c)<\rho_{1} y(x)$ for $x>c$. Thus,

$$
\rho_{1} y(c) / \rho_{1}(x)<y(x) \text { or }-\rho(x) \tilde{c} / \rho_{1}(x)>-p(x) y(x)
$$

where $\tilde{c}=\rho_{1} y(c)>0$. But $y$ is a solution of $(1.1)$, hence

$$
L_{n}[y]<-p(x) \tilde{c} / \rho_{1}(x)
$$

and

$$
\rho_{n} L_{n-1}[y(x)]-\rho_{n} L_{n-1}[y(c)]<-\int_{c}^{x} \frac{p(t)}{\rho_{1}(t)} \hat{c} d t
$$

We have assumed that $L_{n_{1}}[y]$ is negative on $[c, \infty)$ and, by Rolle's Theorem, we know $L_{n_{1}+1}[y], \ldots, L_{n-1}[y]$ are negative on $[c, \infty)$. Then

$$
L_{n-1}[y(x)]=-\frac{\hat{c}}{\rho_{n}(x)} \int_{c}^{x} \frac{p(t)}{\rho_{1}(t)} d t .
$$

After $n+1-n_{1}$ integrations, we find

$$
\rho_{n_{1}} L_{n_{1}}[y(x)]-\rho_{n_{1}} L_{n_{1}-1}[y(c)] \leqq-\hat{c} \int_{c}^{x} \frac{d t}{\rho_{n_{1}+1}} \cdots \int^{t_{n-n_{1}}} \frac{d t_{n-n_{1}}}{\rho_{n}} \int^{t_{n+1-n_{1}}} \frac{p d t_{n+2-n_{1}}}{\rho_{1}}
$$

so that

$$
\lim _{x \rightarrow \infty}\left[\rho_{n_{1}} L_{n_{1}-1}[y(x)]-\rho_{n_{1}} L_{n_{1}-1}[y(c)]\right] \rightarrow-\infty
$$

which is a contradiction. 
In order to show that $L_{n_{1}+1} y$ through $L_{n-1} y$ are positive on $[b, \infty)$, we use a similar argument as that for $L_{1}$ through $L_{n_{1}-1}$ where the assumption that $\chi_{i} \rightarrow \infty$ as $x \rightarrow \infty$ is replaced by the assumption that $\sigma_{j} \rightarrow \infty$ as $x \rightarrow \infty, j \in S$.

Now $L_{n-1} y(x)>0$ on $[b, \infty)$ implies that

$$
\rho_{n-1} L_{n-2}[y(x)]-\rho_{n-1} L_{n-2}[y(c)]>0, \quad c \in(b, \infty),
$$

or

$$
L_{n-2}[y(x)]>\tilde{c} / \rho_{n-1}(x)>0
$$

where $\tilde{c}=\rho_{n-1} L_{n-2}[y(c)]$. Hence, $y(x)>\tilde{c} T_{n-1}(x, c)$. Now

$$
L_{n}[y]=-p y<-\tilde{c} T_{n-1}(x, c)
$$

or

$$
L_{n-1} y(x)-L_{n-1} y(c)<-\tilde{c} \int_{c}^{x} T_{n-1}(t, c) p(t) d t
$$

hence

$$
\lim _{x \rightarrow \infty} L_{n-1}[y(x)]<-\infty
$$

which is a contradiction. Thus $y(x)$ is oscillatory on $[a, \infty)$. The thenrem now follows by Theorem 3 of Mikusinki [4].

For the $2 n$ selfadjoint

$$
\left(r y^{(n)}\right)^{(n)}+p y=0,
$$

we obtain the following theorem of R. Hunt [1] as a corollary.

COROllaRY 3.1. Suppose that

$$
\int^{\infty}\left({ }^{n} \frac{t^{n-2}}{r}\right) p(x) d x=\infty
$$

and

$$
\int^{\infty}\left({ }^{x} I^{n} p\right) \frac{1}{r(x)} d x=\infty
$$

(where ${ }^{x} I^{n}$ denotes the nth iterated integral), then there exists a set of $2 n$ linearly independent solutions of (3.5), with $p>0$ on $[a, \infty)$, as in Theorem 3.1.

COROLlary 3.2. Suppose that

$$
\int^{\infty} x^{n-2} p(x) d x=\infty
$$

then there exists a set of $n$ linearly independent solutions of $y^{(n)}+p y=0$ with $p>0$ on $[a, \infty)$, as in Theorem 3.1 . 


\section{REFERENCES}

1. R. W. Hunt, Oscillation properties of even-order linear differential equations, Trans. Amer. Math. Soc. 115 (1965), 54-61. MR 34 \#2985.

2. W. Leighton and Z. Nehari, On the oscillation of solutions of self-adjoint linear differential equations of the fourth order, Trans. Amer. Math. Soc. 89 (1958), 325-377. MR 21 \#1429.

3. R. A. Leslie, The zeros of solutions to certain linear homogeneous differential equations of even order, Doctoral Dissertation, University of Georgia, Athens, Ga., 1968.

4. J. Mikusinki, Sur l'équation $X^{(n)}+A(t) X=0$, Ann. Polon. Math. 1 (1955), 207-221. MR 19, 141.

5. Z. Nehari, Non-oscillation criteria for $n$th order linear differential equations, Duke Math. J. 32 (1965), 607-615. MR 32 \#4338.

University of Tennessee, Knoxville, Tennessee 37916

Montana State University, Bozeman, Montana 59715 